\title{
The effect of regulating the Wnt signaling pathway on the proliferation and differentiation of spermatogonial stem cells
}

\author{
Leshen Yao ${ }^{1 \#}$, Haiyan Peng ${ }^{2 \#}$, Zhipeng $\mathrm{Xu}^{1}$, Liang Shi ${ }^{1}$, Yan $\mathrm{Li}^{2}$, Yutian Dai ${ }^{1}$ \\ ${ }^{1}$ Nanjing Drum Tower Hospital Clinical College of Nanjing Medical University, Nanjing, China; ${ }^{2}$ Department of Chronic Communicable Disease, \\ Jiangsu Provincial Center for Disease Control and Prevention, Nanjing, China \\ Contributions: (I) Conception and design: L Yao, Y Li, Y Dai; (II) Administrative support: L Yao, H Peng; (III) Provision of study materials or \\ patients: L Yao, Z Xu; (IV) Collection and assembly of data: L Yao, H Peng, Z Xu, L Shi; (V) Data analysis and interpretation: L Yao, H Peng, L Shi; \\ (VI) Manuscript writing: All authors; (VII) Final approval of manuscript: All authors. \\ \#These authors contributed equally to this work. \\ Correspondence to: Yan Li. Department of Chronic Communicable Disease, Jiangsu Provincial Center for Disease Control and Prevention, Nanjing, \\ China. Email: Liyan.nju@163.com; Yutian Dai. Nanjing Drum Tower Hospital Clinical College of Nanjing Medical University, Nanjing, China. \\ Email: 13913957628@163.com.
}

Background: Spermatogonial stem cells and organ engineering research has raised new hope in infertility treatment. Spermatogenesis is a complex physiological process. To observe the proliferation ability and differentiation tendency of mice spermatogonial stem cells (SSCs), to study the effect of regulating the Wnt signaling pathway on the proliferation and differentiation of SSCs, and to provide a valuable basis for the clinical application of SSCs.

Methods: SSCs were isolated and cultured by immunomagnetic separation. Cell surface markers were identified by flow cytometry. Axin 1 was chosen as the target gene to inhibit fibrosis of SSCs by inhibiting the activity of Wnt signaling pathway. Axin-siRNA interference vector was constructed and transfected into spermatogonial stem cells. Cultured SSCs were randomly divided into six groups: control group, SSCs + TGF- $\beta$ group, SSCs + DKK1 group, SSCs + Axin-RNAi group, SSCs + TGF- $\beta+$ DKK1 group, SSCs + TGF- $\beta$ + Axin-RNAi group. Proliferation of SSCs in each group was detected by MTT assay. Immunofluorescence, western blot and real time polymerase chain reaction analysis were used to detect protein expression in the $\mathrm{Wnt} / \beta$ catenin signaling pathways and the molecular markers of fibroblasts in SSCs.

Results: Flow cytometry analysis confirmed that the cultured SSCs were of high purity. MTT assay showed there was no significant difference between Axin-siRNA transfected and non-transfected cells. The proliferation ability was significantly increased in the SSCs + TGF- $\beta$ group, however, it was retarded in SSCs + Axin-RNAi group. The results of immunofluorescence and western blot analysis showed that the expression levels of the Wnt signaling pathway proteins were relatively inhibited after Axin-siRNA was applied. Real-time polymerase chain reaction showed that the expression levels of the molecular markers of fibroblasts were close to the normal control group.

Conclusions: The Axin-siRNA constructed in this study specifically inhibited Wnt/ $\beta$-catenin signal pathway activation, then inhibited the differentiation of SSCs into fibroblasts, which provides a valuable basis for the clinical application of SSCs.

Keywords: Spermatogonial stem cells (SSCs); proliferation; fibroblast differentiation; Wnt signaling pathway; differentiation

Submitted Jun 20, 2020. Accepted for publication Aug 05, 2020.

doi: $10.21037 /$ atm-20-5321

View this article at: http://dx.doi.org/10.21037/atm-20-5321 


\section{Introduction}

In recent years, cells and organ engineering research has raised new hope in infertility treatment $(1,2)$. Spermatogonial stem cells (SSCs), niche in the basal compartment of the seminiferous tubules, is a type of germline stem cell with capacities of self-renewal and differentiation, play an important role in spermatogenesis in males $(3,4)$. Spermatogenesis is a complex physiological process involving multiple molecules and signaling pathways, and it takes more than one month from spermatogonial stem cells to sperm formation, which through meiosis. Spermatogenic failure is a growing problem and is common among reproductive-age men, which could be caused both by diseases influence endocrine abnormalities, such as diabetes, and direct effects on the fidelity and throughput of spermatogenesis.

It is reported that many studies have documented the function of the Wnt and transforming growth factor- $\beta$ (TGF- $\beta$ ) signaling pathways could effect many Physiological Processes, including spermatogenesis $(5,6)$. The $W n t / \beta$-catenin signaling pathway is involved in developmental and physiological processes, such as cell proliferation and differentiation $(7,8)$ and can regulate and activate the production of Wnt6 protein by paracrine of Sertoli cells, and participate in the proliferation regulation of undifferentiated spermatogonia (9).

SSCs can be isolated and cultured in vitro with immunomagnetic beads. SSCs reside along the basement membrane of the seminiferous tubule in close contact with Sertoli cells. Studies have identified how Sertoli cells support SSCs. Recently reports show that Wnt proteins includes the canonical (Wnt/b-catenin and cTnnB1) and non-canonical $\left(\mathrm{Wnt} / \mathrm{Ca}^{2+}\right)$ pathways. A less studied Wnt pathway is the Wnt-JnK pathway which plays a crucial role in stem cell morphogenesis, cell proliferation, differentiation and migration $(10,11)$.

The $\beta$-catenin complex, composed mainly of adenomatous polyposis coli (APC), axin, glycogen synthase kinase- $3 \beta$ (GSK-3 $\beta$ ), and casein kinase 1 , has been identified as crucial for the maintenance of the stem-like properties of stem cells and for clinic treatment resistance. In this study, we have investigated the proliferation ability and differentiation tendency of mice SSCs. Furthermore, we have studied the effect of regulating the Wnt signaling pathway on the proliferation and differentiation of SSCs, to provide a basis for the clinical application of SSCs.

We present the following article in accordance with the
ARRIVE reporting checklist (available at http://dx.doi. org/10.21037/atm-20-5321).

\section{Methods}

\section{Isolation and culture of SSCs}

SPF C57BL/6 male mice (5-10 days) were purchased from Nanjing Slack Laboratory Animal Co., Ltd. The mice were put on a 12-hour dark/light cycle with chow and water provided on time. SSCs were collected from the mice and purified according to an existing protocol $(12,13)$. In brief, the mice were killed by carbon dioxide suffocation. The testis was collected under aseptic conditions, then the seminiferous tubules were washed five times with phosphate-buffered saline (PBS). Following two-step enzymatic digestion as described by Ko et al. [2010] (14) the suspensions of testicular cells were collected. THY1positive cells were enriched by magnetic activated cell separation (MACS). Cells were re-suspended in stem cell culture medium (Invitrogen, CA, USA), supplemented with $10 \%$ stem cell fetal bovine serum (FBS), $5 \%$ of $50 \mu \mathrm{g} / \mathrm{mL}$ penicillin, and streptomycin, and seeded at a density of $1 \times 10^{6}$ cells in $25 \mathrm{~cm}^{2}$ cells culture flasks. They were maintained at $37{ }^{\circ} \mathrm{C}$ in a $95 \%$ humidified atmosphere and $5 \% \mathrm{CO}_{2}$ (Thermol, RBT, Germany). The medium was changed 4 days after seeding, then every 5 days until the cells reached approximately $85 \%$ confluence. The cells were passaged by $0.25 \%$ pancreatic enzyme. Cells of passages 3-9 were used for experiments. All animal experiments were performed in accordance with the guidelines for animal care and the protocol was approved by the Committee on the Ethics of Animal Experiments of Jiangsu Provincial Center for Disease Prevention and Control (approval no. JSJK/JL-161).

\section{Surface molecular phenotype analysis of SSCs}

The molecular phenotype of the mice SSCs was identified by flow cytometry (FACSCalibur, BD, NY, USA). Expression of the surface markers CDH1, ID4, NANOS3, CD44, CD 31 and CD45 were detected. $1 \times 10^{6}$ SSCs (Passage 3 and 10) were incubated with non-fluorescent labeling primary antibodies (rabbit anti-mouse CDH1, ID4, NAN0S3, CD44, CD31 and CD45) for 2 hours at room temperature. Then, cells were incubated by a secondary antibody [goat anti-rabbit Alexa Fluor 488 (Invitrogen Life Science, NY, USA)] for 1 hour at room temperature in 
darkness. SSCs were washed 3 times by PBS and detected using flow cytometry (BD, NJ, USA). Data were analysed using Paint-A-Gate and FlowJo software (TreeStar Inc. San Carlos, CA).

\section{The interference sequence siRNA of the Wnt signaling patbway key molecular Axin gene}

The siRNA-expressing construct of the Axin gene was generated as follows: according to the Axin gene sequence (NCBI: nm_009733), Axin gene siRNA interference sequences were designed by Ambion siRNA Target Finder software, and 5'-TCCCACTTTGAATGAGGA TGAACTCGAG TTCATCCTCATTCAAAGTGGG TTTTTTC-3' was selected, chemically synthesized, annealed, and digested with EcoR I/XhoI, and ligated into the same restriction sites in the green fluorescent protein (GFP)-labeled lentivirus vector pGC-LV expression vector (GV118). DNA sequencing was applied to identify the transformant. A random siRNA sequence (TTCTCCGAACGTGTCACGT) was used as a control for Axin siRNA.

Lenti-NC and Lenti-Axin-si virus were amplified by $293 \mathrm{~T}$ cells with ViraPower Packaging Mix and Lipofectamine 2000 reagent, according to manufacturer instructions. In brief, $293 \mathrm{~T}$ cells were transfected with Lenti-Axin-si virus. 48 hours after transfection, the supernatant was collected and passed through $0.45 \mu \mathrm{m}$ filters and concentrated. The viral supernatant was added into target SSCs cells at multiplicity of infection $(10,100$, $200 \mathrm{MOI}$ ) with $5 \mathrm{~g} / \mathrm{mL}$ polybrene to obtain stable LentiAxin-si virus transfected cells. Transfection positive cells were detected by fluorescence microscopy. Expression of the Axin protein was detected by Western-blotting.

\section{Cell viability and proliferation after Lenti-Axin-si transfection was detected by MTT assay}

MTT [3-(4,5-Dimethylthiazol-2-yl)-2,5-diphenyltetrazolium bromide] assay was performed from the first day to the tenth day after Lenti-Axin-siRNA virus transfection. In brief, the SSCs were cultured in 96-well plates. Cells were incubated with $25 \mu \mathrm{L}$ MTT solution $(5 \mathrm{mg} / \mathrm{mL}$ ) for 4 hours at room temperature. Supernatant was discarded and $100 \mathrm{~L}$ DMSO was added to dissolve the formazan precipitate for half an hour at $37^{\circ} \mathrm{C}$. Optical density was then measured at $570 \mathrm{~nm}$ with an automatic microplate spectrophotometer (SpectraMax 190, Sunnyvale, USA).

\section{Effect on SSCs differentiation by the Wnt/B-catenin signaling pathway}

\section{SSCs grouping}

Cultured SSCs were divided into six groups: control group (normal SSCs), SSCs + TGF- $\beta$ group (TGF- $\beta 0.5 \mathrm{ng} / \mathrm{mL}$ was added to the culture medium to induce SSCs to differentiate into fibroblasts), SSCs + DKK1 group (positive control, Wnt/ $\beta$-catenin signaling pathway inhibitor DKK1 $20 \mathrm{ng} / \mathrm{mL}$ was added to the culture medium), SSCs + AxinRNAi group (Wnt/ $\beta$-Catenin signal pathway specific interference virus Axin-RNAi $10^{-3}$ was added to the culture medium to decrease Axin expression to inhibit the Wnt signal pathway), SSCs + TGF- $\beta$ + DKK1 group $(0.5 \mathrm{ng} / \mathrm{mL}$ TGF $-\beta$ and $20 \mathrm{ng} / \mathrm{mL}$ DKK1 were both added to the culture medium), SSCs + TGF- $\beta$ + Axin RNAi group $\left(0.5 \mathrm{ng} / \mathrm{mL}\right.$ TGF- $\beta$ and Axin-RNAi $10^{-3}$ were both added to the culture medium).

\section{Induce SSCs differentiate into fibroblasts}

TGF- $\beta$ was added to the culture medium to induce SSCs to differentiate into fibroblasts. Second to 5 th generation mesenchymal stem cells were chosen for observation. Cells were subcultured in low glucose DMEM with $10 \%$ FBS and $0.5 \mathrm{ng} / \mathrm{mL}$ TGF- $\beta$. The SSCs were inoculated in the culture plate at $5 \times 10^{5} / \mathrm{mL}$ density at $37{ }^{\circ} \mathrm{C}, 5 \% \mathrm{CO}_{2}$.

\section{Specific inhibit $\mathrm{Wnt} / \beta$-catenin signaling pathways of SSCs}

Axin-RNAi was applied to decrease the expression of Axin protein, and specifically inhibit the $\mathrm{Wnt} / \beta$-catenin signaling pathway of the SSCs. Second to 5th generation SSCs were subcultured in low glucose DMEM with $10 \%$ FBS and Axin-RNAi (100 MOI). DKK1 was chose as the positive control. The SSCs were inoculated in the culture plate at $5 \times 10^{5} / \mathrm{mL}$ density at $37^{\circ} \mathrm{C}, 5 \% \mathrm{CO}_{2}$.

\section{Induce SSCs to differentiate into fibroblasts combine with specific inhibition of Wnt/ $\mathrm{W}$-catenin signaling pathway}

SSCs were induced to differentiate into fibroblast by TGF- $\beta$. Axin-RNAi was used to inhibit the $W n t / \beta$-catenin signal pathway. Second to 5 th generation SSCs were subcultured in low glucose DMEM with $10 \%$ FBS and $0.5 \mathrm{ng} / \mathrm{mL}$ TGF- $\beta$ + Axin-RNAi (100 MOI). DKK1 was chose as the positive control. The SSCs were inoculated in the culture plate at $5 \times 10^{5} / \mathrm{mL}$ density at $37{ }^{\circ} \mathrm{C}$, $5 \% \mathrm{CO}_{2}$. 


\section{Cell viability and proliferation detection}

Viability and proliferation of SSCs was detected by MTT assay from the 1 st to 10 th day after treatment. In brief, SSCs cells were cultured in $96-$ well plates. The cells were incubated with $25 \mu \mathrm{L}$ MTT solution $(5 \mathrm{mg} / \mathrm{mL})$ for 4 hours at room temperature, supernatant was discarded and $100 \mu \mathrm{L}$ DMSO was added to dissolve the formazan precipitate for half an hour at $37^{\circ} \mathrm{C}$. Optical density was measured at $570 \mathrm{~nm}$ with an automatic microplate spectrophotometer (SpectraMax 190, Sunnyvale, USA).

\section{The key protein of Wnt/B-catenin signaling patbways expression}

Protein $\beta$-catenin, CD14, p-ERK and p-Jun N-terminal kinase (p-JNK) expression in $\mathrm{Wnt} / \beta$-catenin signaling pathways of SSCs after treatment for each group was detected by cell immunofluorescence. Briefly, a further three generations of SSCs were cultivated according to the grouping method. SSCs were fixed in precool methanol for 20 minutes. Sealed non-specific protein in $1 \%$ bovine serum albumin (BSA) for 1 hour. Cells were then incubated in diluting primary antibodies for 2 hours at $37^{\circ} \mathrm{C}$ (rabbit anti-mouse $\beta$-catenin, CD14, p-ERK and p-JNK) (Abcam Inc., Cambridge, MA), followed by the secondary antibody for 1 hour at $37^{\circ} \mathrm{C}$ [fluorescein labeled antibody to rabbit $\operatorname{IgG}(\mathrm{H}+\mathrm{L}) ; 1: 100]$. The cell nucleus was incubated with diamidino-phenyl-indole for 10 minutes at room temperature. Cells were washed 5 times by PBST between each step. Fluorescence was detected and recorded by the inverted fluorescence microscope.

\section{The expression of fibroblast surface markers in SSCs}

The expression of fibroblast surface markers vimentin, $\alpha$-smooth muscle actin ( $\alpha$-SMA) and the anti-fibroblast antibody, TE-7 in SSCs was analysed by western blotting. Three further generations of SSCs were cultivated according to the grouping method. Then, cells were lysed in ice-cold extraction buffer containing protease inhibitor cocktail (Roche) and centrifuged at 10,000 rpm for 10 minutes. The protein concentration in the supernatant was determined using Bradford assays. Proteins were separated using $4 \%$ concentrated gel and $10 \%$ sodium dodecyl sulfate polyacrylamide gel electrophoresis and electrophoretically transferred to polyvinylidene fluoride membranes. The membranes were sealed at $37{ }^{\circ} \mathrm{C}$ for
1 hour in PBST and $5 \%$ (wt/vol) non-fat milk. Membranes were then incubated in primary antibody at a 1:500 dilution in PBSTM for 2 hours at room temperature and the appropriate horseradish peroxidase (HRP)-conjugated anti-species secondary antibodies (Boster, Wuhan, China) for 1 hour at room temperature then washed in PBSTM as described above. Immunoreactive protein bands were detected using an Odyssey Scanning System (LI-COR, Inc., Lincoln, NE, USA). Ratios for the protein of interest (POI) were expressed relative to glyceraldehyde 3-phosphate dehydrogenase in the same sample as a loading control. The primary antibodies rabbit anti-vimentin, $\alpha$-SMA, TE-7, and glyceraldehyde 3-phosphate dehydrogenase were obtained from Santa Cruz (USA).

\section{mRNA expression of fibroblast markers in SSCs}

mRNA expression level of the fibroblast surface markers vimentin, $\alpha$-SMA and TCF were analysed by real-time reverse transcription polymerase chain reaction (qRT-PCR). Primers and probes are shown in Table 1.

\section{Statistical analysis}

Each experiment was performed in sextuplicate and repeated a minimum of three times. Results are expressed as mean \pm SEM. All calculations and statistical analyses were performed using SPSS 18.0. $T$-tests and one-way ANOVA followed by Tukey honest significance test were used to analyse data differences. $\mathrm{P}<0.05$ was considered statistically significant.

\section{Results}

\section{SSCs culture in vitro}

The morphology of cultured SSCs was observed by the microscope. Some cells adhered to the bottom of the flasks after 3 days. Seven days later, colonies of SSCs, including single and double spheres, and clusters, grew uniformly into spiral shape or long shuttle type. After 14 days, the cells were a mixed $80 \%$ fusion. After 3 generations, the SSCs grew faster, and in a unified form (Figure 1).

\section{Surface molecular phenotype identify of SSCs by FACS}

Flow cytometry analysis showed that the 3 rd generation of SSCs expressed CDH1, ID4, NAN0S3 and CD44, but did not express CD31 or CD45 (Figure 2), which was consistent 
Table 1 Primers and probes to analyse the mRNA expression level of fibroblast markers and $\mathrm{Wnt} / \beta$-catenin signaling molecules in spermatogonial stem cells

\begin{tabular}{|c|c|}
\hline Gene name & Probe and primer sequence $\left(5^{\prime}-3^{\prime}\right)$ \\
\hline$M u s-\alpha-S M A-F P$ & CCTGACGCTGAAGTATCCGATAG \\
\hline$M u s-\alpha-S M A-R P$ & TTTTCCATGTCGTCCCAGTTG \\
\hline$M u s-\alpha-S M A-P R O B E$ & VIC-ACACGGCATCATCA-BHQ2 \\
\hline$M u s-T C F-F P$ & CGCCTGAGGGTCCGAGATA \\
\hline$M u s-T C F-R P$ & GCACCATACGGCCAAGCT \\
\hline Mus-TCF-PROBE & VIC-CGCGACCCGAATTGAGA-BHQ2 \\
\hline$M u s-G S K-3 \beta-F P$ & СССTCTGGCCACCATCCT \\
\hline$M u s-G S K-3 \beta-R P$ & СССТCTGGCCACCATCCT \\
\hline$M u s-G S K-3 \beta-P R O B E$ & VIC-CCСTCCACATGCTC-BHQ2 \\
\hline$M U S-G A P D H-F P$ & CATGGCCTTCCGTGTTCCTA \\
\hline$M U S-G A P D H-R P$ & GCGGCACGTCAGATCCA \\
\hline MUS-GAPDH-PROBE & VIC-CCCCAATGTGTCCGTC-BHQ2 \\
\hline
\end{tabular}

with the characteristics of surface molecular markers of SSCs. The purity of SSCs was approximately $95 \%$. However, for the 10th generation of SSCs, only CD44 was expressed (Figure 3). The experiments were performed in triplicate.

\section{The observation of SSCs cells after transfection}

\section{Fluorescence microscopy observation}

The Axin-RNAi transfection positivity of SSCs cells was observed by fluorescence microscopy, and the proliferation of SSCs was detected by MTT assay after transfection. We found the transfection positivity of SSCs in the 100 and 200 MOI groups were high than in the $10 \mathrm{MOI}$ group, and no obvious pathological changes were found among the cells in the control, 10 and 100 MOI transfected groups (Figure 4).

\section{Western blotting analysis}

Western blotting results showed that after 48 hours the expression of the Axin protein in the 100 and 200 MOI
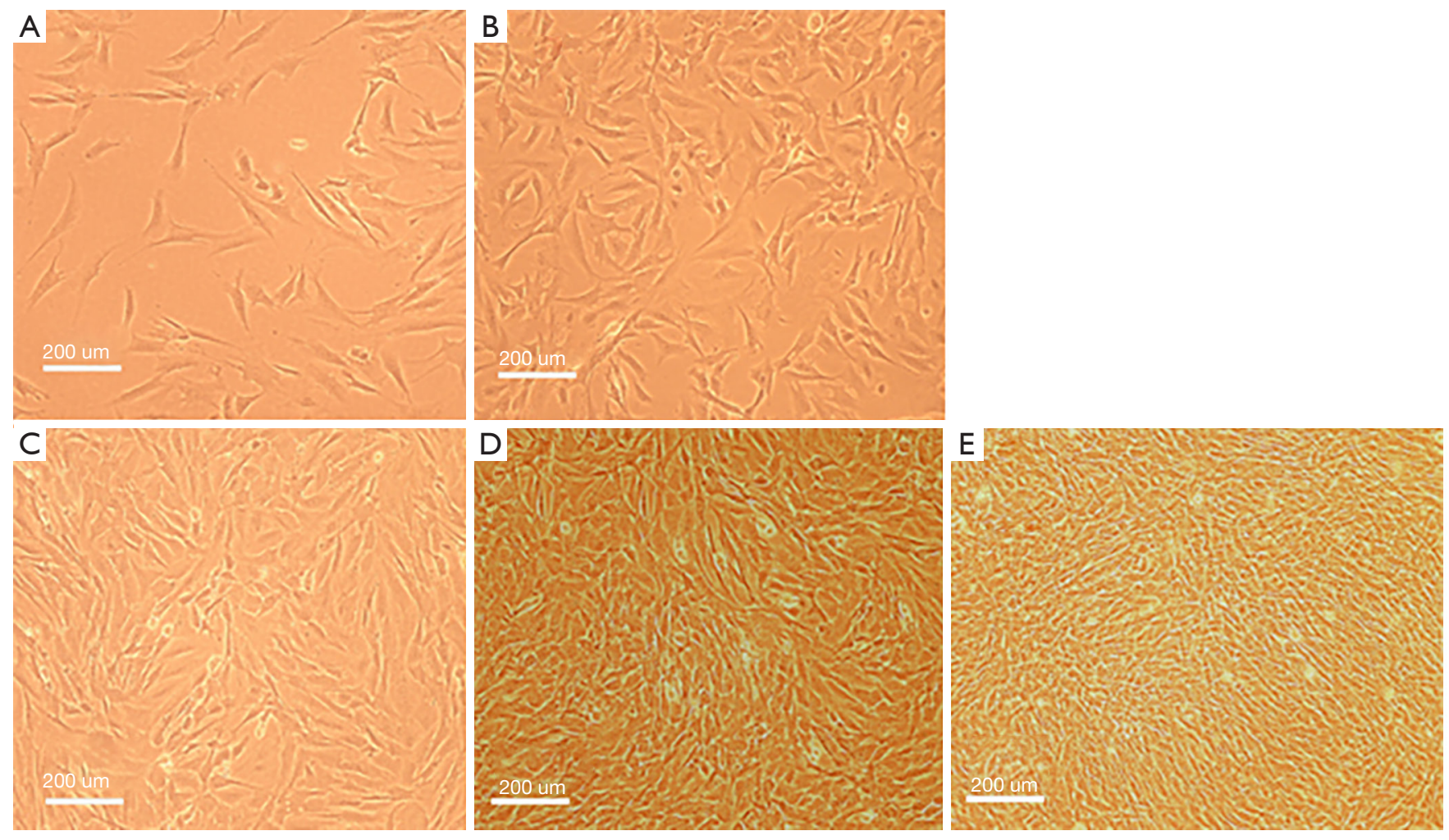

Figure 1 Microscopy images of generation and sub-cultured mice SSCs in vitro $(\times 20)$. After $3^{\text {rd }}$ generation of culture, SSCs grew fast into a unified form, while after $11^{\text {th }}$ generation of culture, SSCs grew faster and lose the normal unified form. (A) 7 days of original cultured SSCs; (B) SSCs original generation and cultured 14 days; (C) $3^{\text {rd }}$ generation of SSCs; (D) $10^{\text {th }}$ generation of SSCs; (E) $11^{\text {th }}$ generation of MSCs. SSCs, spermatogonial stem cells. 

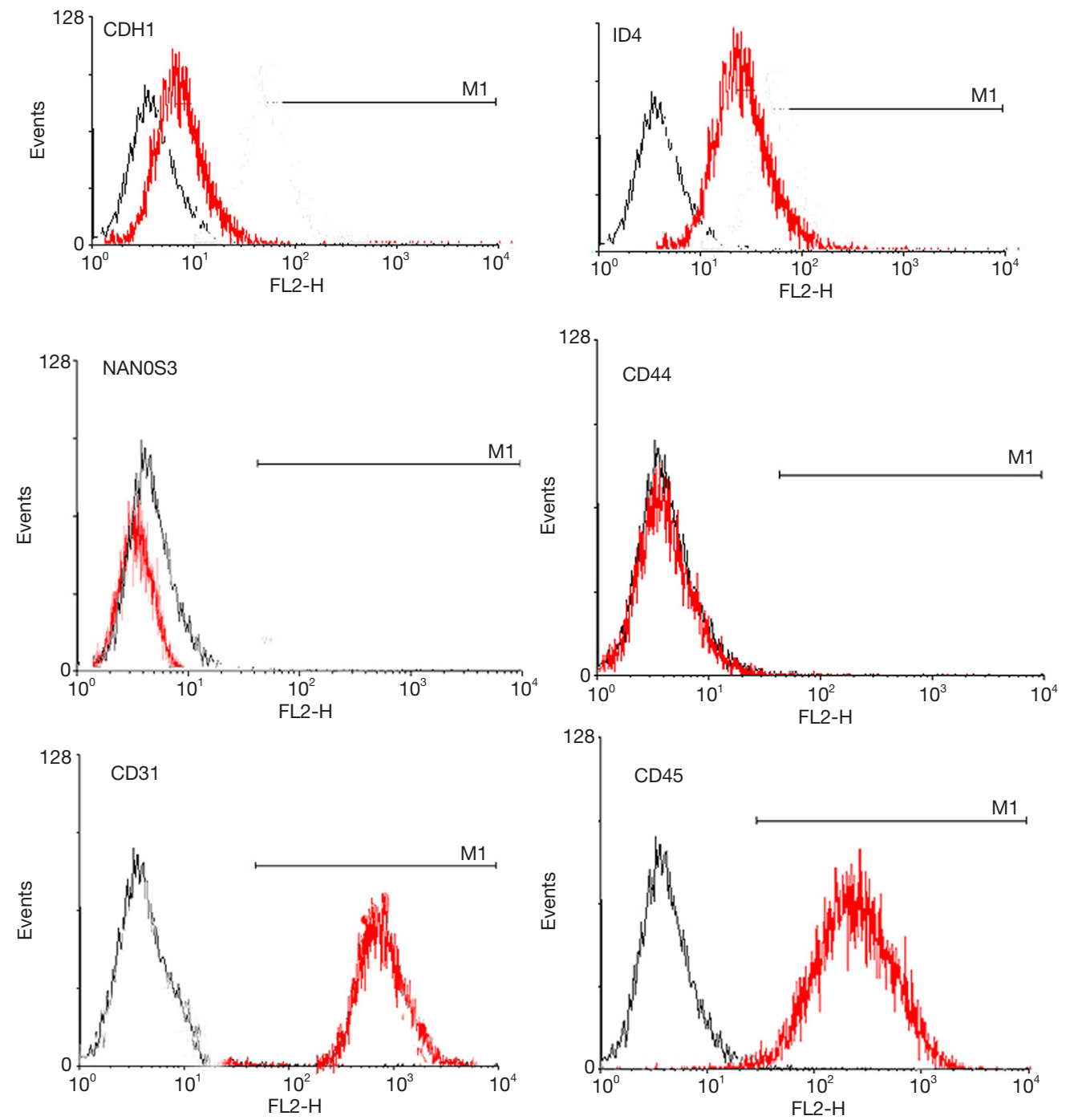

Figure 2 Flow cytometry identified the surface markers of 3rd generation SSCs. SSCs expressed CDH1, ID4, NAN0S3 and CD44, but did not express CD31 or CD45. X-axis = fluorescence intensity, Y-axis = cell counts. SSCs, spermatogonial stem cells; CD, cluster of differentiation.

transfected groups were significantly decreased compared with those in the control and 10 MOI transfected groups (Figure 5).

\section{MTT detection}

MTT results showed there was no significant difference in the proliferation ability of SSCs in the 10 and 100 MOI Axin-RNAi transfected groups compared with the control group in the 10 days post transfection, while the proliferation ability of SSCs in the 200 MOI transfected group was decreased significantly compared with the control group (Figure 6).

These indicated that 100 MOI Axin-RNAi virus solution transfection could reduce the expression of Axin protein, and had no significant effect on the proliferation ability and cell activity of SSCs cells. 

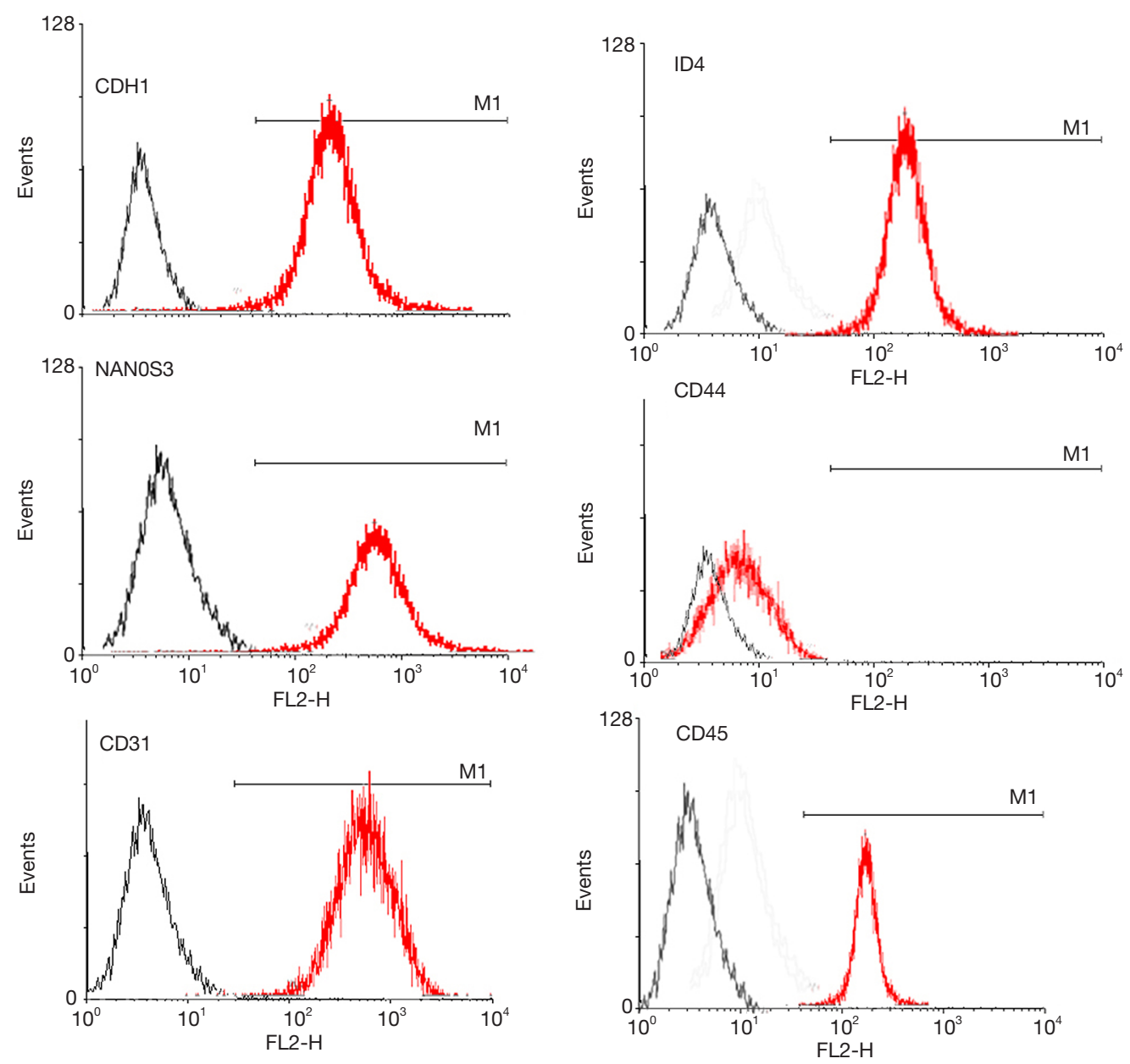

Figure 3 Flow cytometry identified the surface markers of 10th generation SSCs. SSCs expressed CD44, but did not express CDH1, ID4, NAN0S3, CD31 or CD45. X-axis = fluorescence intensity, Y-axis = cell counts. SSCs, spermatogonial stem cells; CD, cluster of differentiation.
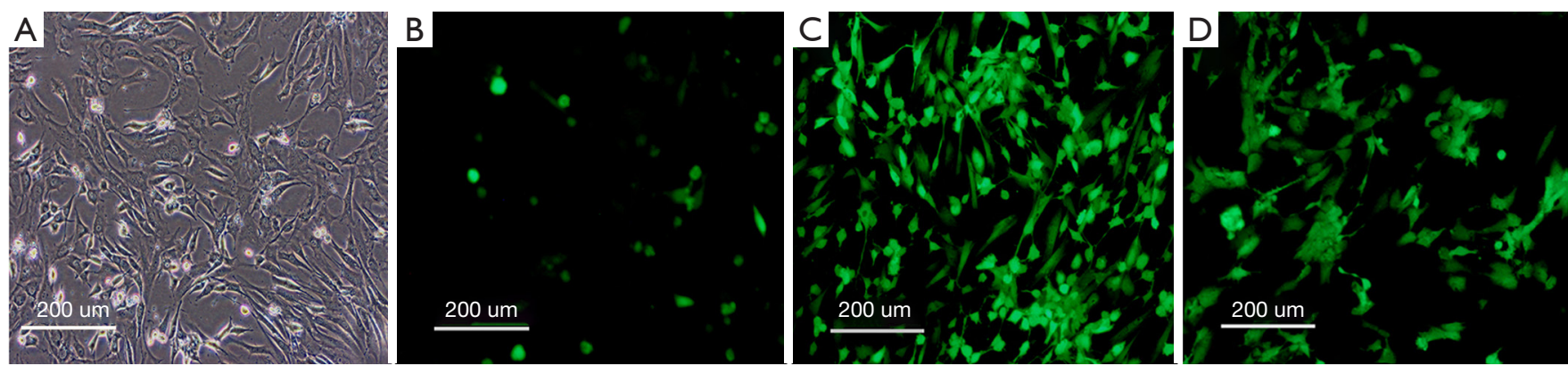

Figure 4 Inverted phase and fluorescence microscopy images of SSCs after Axin-RNAi transfection. (A) 100 MOI Axin-RNAi transfection group observed by inverted phase contrast microscope; (B) 10 MOI Axin-RNAi transfection group observed by fluorescence microscope; (C) 100 MOI Axin-RNAi transfection group observed by fluorescence microscope; (D) 200 MOI Axin-RNAi transfection group observed by fluorescence microscope. 


\section{Influence of Lenti-Axin-si transfection on SSCs differentiation}

MTT assay was used for detecting the proliferation differences in the different groups of SSCs. It was found that TGF- $\beta$ could influence the proliferation ability of SSCs. The proliferation ability was significantly promoted in SSCs + TGF- $\beta$ group, with the obviously random arrangement of cells. However, in SSCs + Axin-RNAi group, proliferation activity of SSCs was retarded, maintaining uniform arrangement, long spindle and swirling cell morphology. There was no significantly difference between the SSCs +

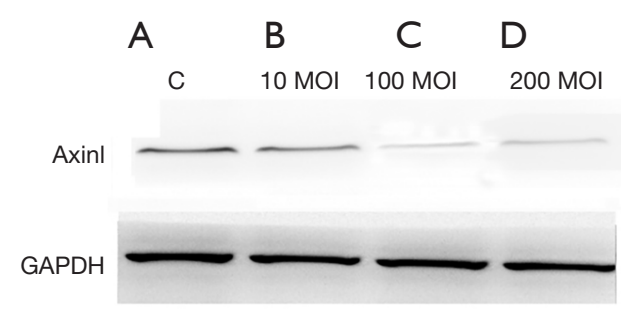

Figure 5 Western blotting analysed the expression of Axin protein in different groups of SSCs after Axin-RNAi transfection for 48 hours. (A) Control group; (B) 10 MOI Axin-RNAi transfected group; (C) 100 MOI Axin-RNAi transfected group; (D) 200 MOI Axin-RNAi transfected group. SSCs, spermatogonial stem cells.
Axin-RNAi group and the SSCs + DKK1 group, also shown by MTT test (Figure 7).

\section{Protein expression level of Wnt/B-catenin signaling pathway in SSCs}

Protein $\beta$-catenin, CD14, p-ERK and p-JNK expression of $W n t / \beta$-catenin signaling pathways in the different groups of SSCs after treatment were detected by cell immunofluorescence.

When TGF- $\beta$ was added to the culture, the Wnt/ $\beta$-catenin signaling pathway was highly activated. In the SSCs + TGF- $\beta$ group, $\beta$-catenin, CD14, p-ERK and p-JNK protein expression increased significantly 3 days later. The expression of these proteins in the SSCs + TGF- $\beta+$ DKK1 group and the SSCs + Axin-RNAi group were reduced in comparison with MSCs + TGF- $\beta$ (Figure 8).

\section{Expression of fibroblast surface markers}

The expression of fibroblast surface markers (vimentin, $\alpha$-smooth muscle actin ( $\alpha$-SMA) and TE- 7 ) in different groups of SSCs were analysed by western blotting. The results showed that the expression levels of vimentin, $\alpha$-SMA and TE-7 were up-regulated in the SSCs + TGF- $\beta$ group, while in the SSCs + TGF- $\beta+$ Axin-RNAi

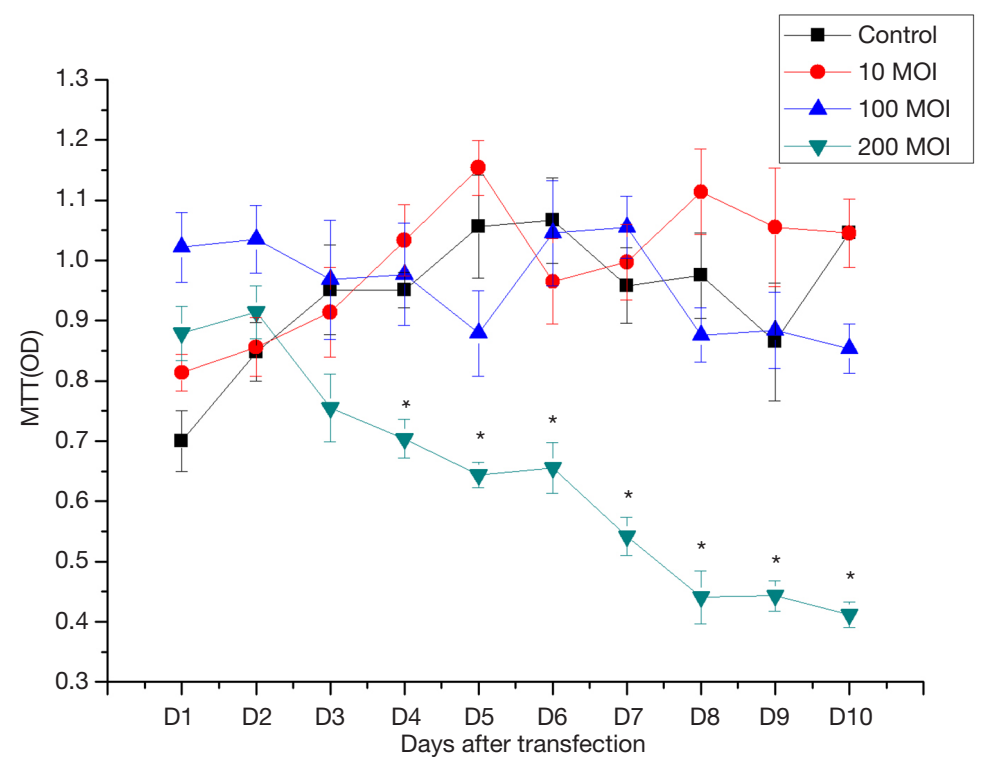

Figure 6 Proliferation activity diagrams of SSCs after different concentrations of Axin-RNAi transfection by MTT detection. Data are shown as mean $\pm \mathrm{SD} .{ }^{*}$, response significantly different to the control $(\mathrm{P}<0.05)$. SSCs, spermatogonial stem cells. 


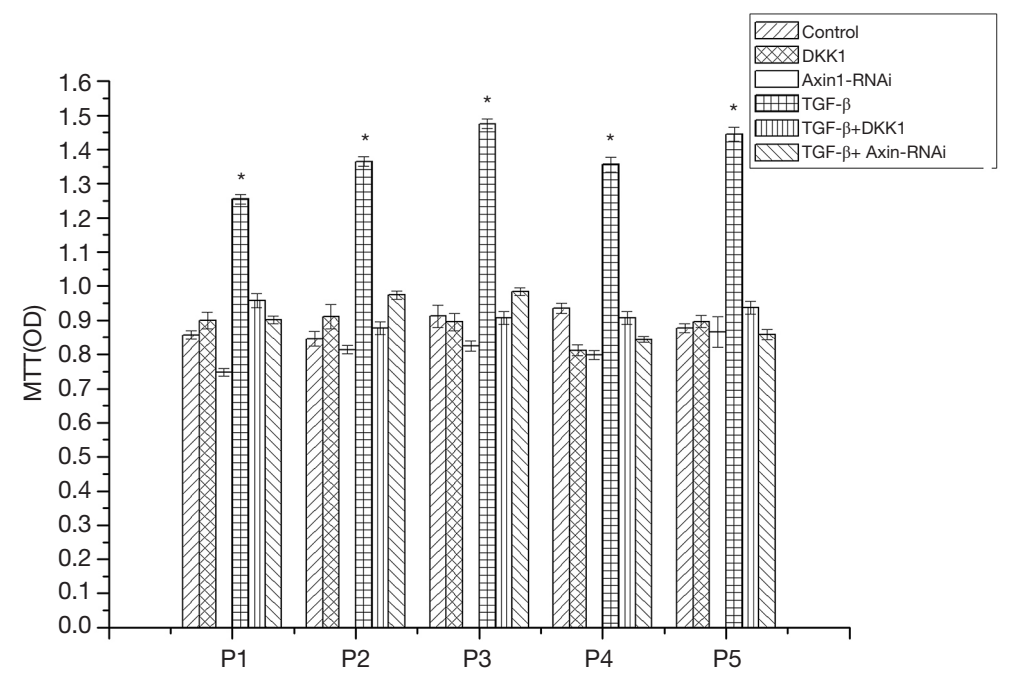

Figure 7 MTT detection of proliferation activity in different groups of SSCs after treatment. Data are shown as mean \pm SD. *, response significantly different to the control $(\mathrm{P}<0.05)$. SSCs, spermatogonial stem cells.

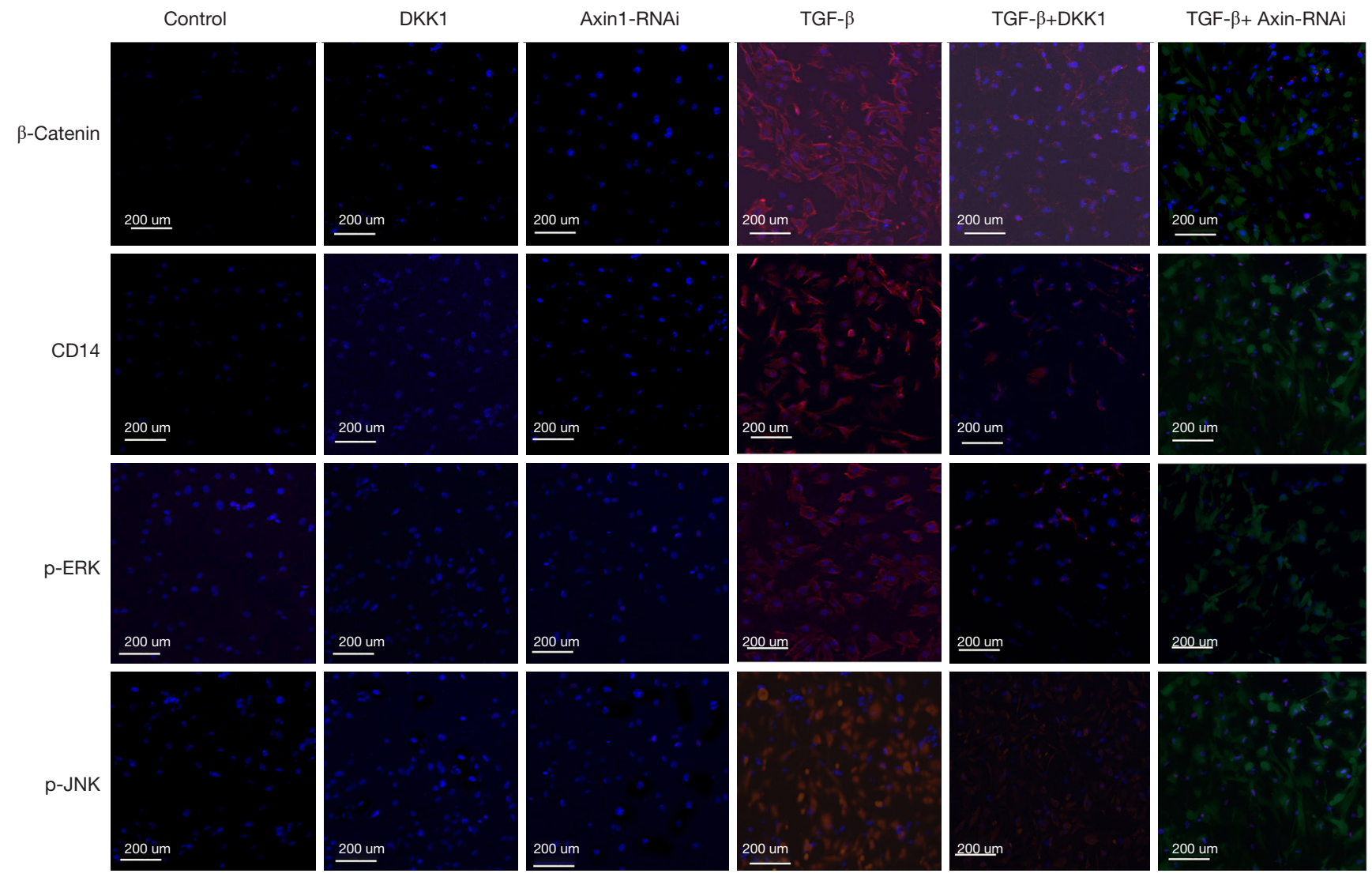

Figure 8 Immunofluorescence observation of protein expression of Wnt/ $\beta$-catenin signaling pathway in different groups of SSCs after treatment. SSCs, spermatogonial stem cells. 
group the expression levels were close to the control group (Figure 9).

\section{$m R N A$ expression of fibroblast markers in SSCs}

mRNA expression level of fibroblast surface markers vimentin, $\alpha$-SMA and TCF were analysed by qRT-PCR. The results showed that they were significantly increased in MSCs of the SSCs + TGF- $\beta$ group compared with the SSCs + TGF- $\beta$ + Axin-RNAi group, in which expression levels of fibroblast surface markers were close to the control group (Figure 10).

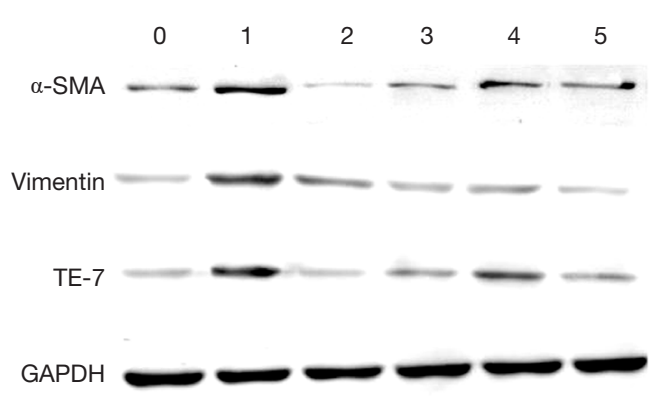

Figure 9 Western blotting analysis of fibroblast surface marker expression in different groups of SSCs after treatment. SSCs, spermatogonial stem cells. C: Control group; 1 : SSCs + TGF- $\beta$ group; 2: SSCs + DKK1 group; 3: SSCs + Axin-RNAi group; 4: SSCs + TGF- $\beta$ + DKK1; 5 : SSCs + TGF- $\beta$ + Axin RNAi group.

\section{Discussion}

Spermatogenesis is a series of complex processes of sequential cell proliferation and differentiation in male testis, affected by many crucial proteins and signal pathways $(15,16)$. Because SSCs produce a large number of progenitors, they are important in the spermatogenesis process (17). In a previous study we found that the use MSCs could lead to a significant increase in the expression of fibroblast growth factor and TGF- $\beta$ both in serum and testis tissue of mice, which may lead to fibrosis of cells and tissues. Previous studies have shown that the mitogenactivated protein kinases (MAPK) regulates and activates the Wnt signaling pathway, could influencing spermatogonia via the non-canonical Wnt pathway.

The results of this study indicate that TGF- $\beta$ can activate the $W n t / \beta$-catenin signaling pathway by upgrading the expression of some key proteins in the Wnt signaling pathway. Subsequently, the proliferation of SSCs was significantly increased, but the majority of these cells proved to differentiate into fibroblasts. These findings are similar to research by Yeh et al. which reported that Wnt3a can activate the $\beta$-catenin pathway in a subset of cluster cells (18). However, SSCs show a tendency to differentiate under this environment. Our results indicate that the activation of canonical $W n t / \beta$-catenin signal pathway could induce the SSC proliferation in vitro, and SSC might be present a tendency of fibrosis by influencing spermatogonia via the canonical Wnt pathway.

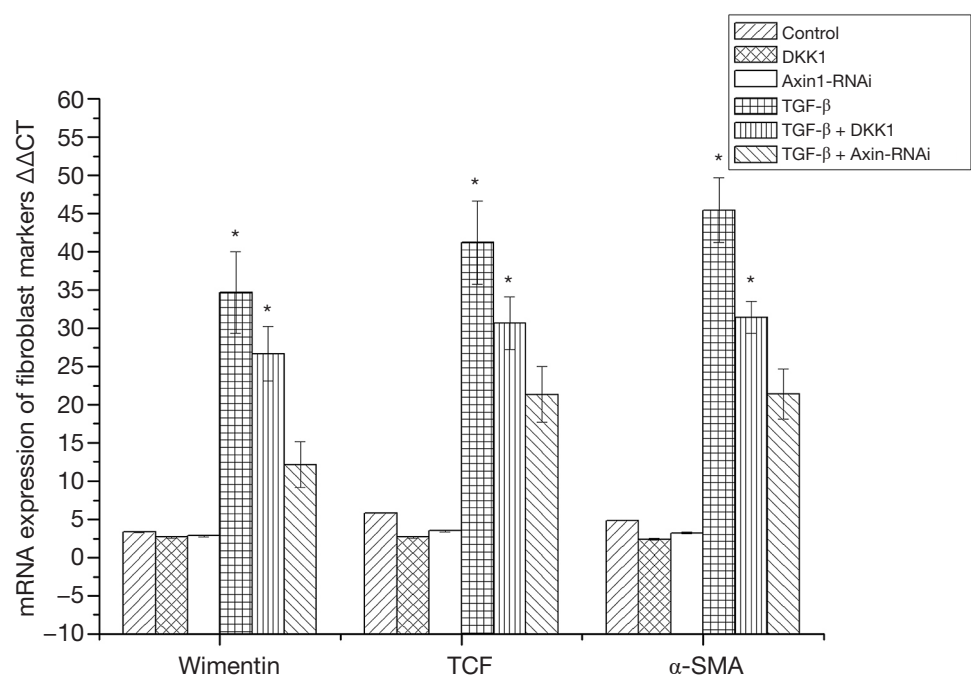

Figure 10 qRT-PCR analysis of fibroblast surface marker expression in different groups of SSCs after treatment. * response significantly different to the control $(\mathrm{P}<0.05)$. SSCs, spermatogonial stem cells. 
It has been reported that the Wnt signaling pathway, including Wnt ligands, Wnt protein receptors and downstream transcription factors, plays an important role in cell proliferation and differentiation $(19,20)$. Our results showed that TGF- $\beta$ can activate the $\mathrm{Wnt} / \beta$-catenin signaling pathway by upgrading the expression of the key proteins $\beta$-catenin, CD14, p-ERK and p-JNK, which leads to upgraded expression of the fibroblast surface markers vimentin, $\alpha$-SMA, TCF and TE-7, which subsequently include differentiation of SSCs into fibroblasts. These results were similar to those in the study by $\mathrm{HE}$, which stated that PKC is specially involved in the Wnt5ainduced differentiation of chicken embryonic stem cells and SSCs (21). Therefore, how to regulate the differentiation direction of SSCs, maintain their normal cell characteristics, and avoid the tendency of fibroblast differentiation, could be a valuable focus for SSCs research.

We speculated that some signals of canonical Wnt pathway might be the key molecules to regulate the differentiation direction of SSCs. In order to confirm this inference, we constructed an interference sequence siRNA of Wnt signaling pathway key molecular Axin gene and transfected it into SSCs, to regulate the activity of $W n t / \beta$-catenin signaling pathway by down-regulate the expression of Axin. Our results proved that once Axin-RNAi was applied, the Wnt signaling pathway in SSCs could be relatively inhibited. Immunofluorescence detection showed that the expression levels of the Wnt signaling pathway molecules such as $\beta$-Catenin, TCF, GSK-3 $\beta$, Axin, and JNK tended to be normal in SSCs. Western blot and real-time PCR were used to detect vimentin, $\alpha$-SMA, TCF and TE-7. We found that the expression levels of fibroblast markers vimentin, $\alpha$-SMA, TCF and TE-7 were close to the control group. The canonical Wnt pathway is referred to as the $\beta$-catenin pathway. The key effect of this pathway including $\beta$-catenin, Axin, and JNK which are commonly found in the cytoplasm of cells. On activation of the canonical Wnt pathway, the activated key protein could regulate the transcription of targeted genes. In our present study we deduced the regulation of canonical Wnt pathway could influence the differentiation direction of SSCs. This study is a mechanism study at the cellular level, and further study on its animal level and human mechanism is needed.

\section{Conclusions}

In summary, our study suggests that the $\mathrm{Wnt} / \beta$-catenin signaling pathway contributes to SSC fibroblast differentiation. In addition, we showed that an AxinRNAi interference virus could block $W n t / \beta$-catenin signal pathway activation by inhibiting Axin protein expression. Specific inhibition of the $\mathrm{Wnt} / \beta$-catenin signaling pathway can inhibit the differentiation of SSCs into fibroblasts and be valuable for clinical application of SSCs.

\section{Acknowledgments}

Funding: None.

\section{Footnote}

Reporting Checklist: The authors have completed the ARRIVE reporting checklist. Available at http://dx.doi. org/10.21037/atm-20-5321

Data Sharing Statement: Available at http://dx.doi. org/10.21037/atm-20-5321

Conflicts of Interest: All authors have completed the ICMJE uniform disclosure form (available at http://dx.doi. org/10.21037/atm-20-5321). The authors have no conflicts of interest to declare.

Ethical Statement: The authors are accountable for all aspects of the work in ensuring that questions related to the accuracy or integrity of any part of the work are appropriately investigated and resolved. All animal experiments were performed in accordance with the guidelines for animal care and the protocol was approved by the Committee on the Ethics of Animal Experiments of Jiangsu Provincial Center for Disease Prevention and Control, (approval no. JSJK/JL-161).

Open Access Statement: This is an Open Access article distributed in accordance with the Creative Commons Attribution-NonCommercial-NoDerivs 4.0 International License (CC BY-NC-ND 4.0), which permits the noncommercial replication and distribution of the article with the strict proviso that no changes or edits are made and the original work is properly cited (including links to both the formal publication through the relevant DOI and the license). See: https://creativecommons.org/licenses/by-nc-nd/4.0/.

\section{References}

1. Yamasaki S, Yoshida S, Kato K, et al. Effects of stem 
cell transplantation in patients with peripheral T-cell lymphoma not otherwise specified and angioimmunoblastic T-cell lymphoma. Int J Hematol 2020;112:74-83.

2. Mohseni M, Shojaei S, Mehravi B, et al. Natural polymeric nanoparticles as a non-invasive probe for mesenchymal stem cell labelling. Artif Cells Nanomed Biotechnol 2020;48:770-6.

3. Sharma A, Kumaresan A, Mehta P, et al. Successful transplantation of transfected enriched buffalo (Bubalus bubalis) spermatogonial stem cells to homologous recipients. Theriogenology 2020;142:441-9.

4. Zhao H, Li T, Yang H, et al. The effects of growth factors on proliferation of spermatogonial stem cells from Guangxi Bama mini-pig. Reprod Domest Anim 2019;54:1574-82.

5. Kanatsu-Shinohara M, Yamamoto T, Toh H, et al. Aging of spermatogonial stem cells by Jnk-mediated glycolysis activation. Proc Natl Acad Sci U S A 2019;116:16404-9.

6. Paucarmayta A, Taitz H, Casablanca Y, et al. TGF- $\beta$ signaling proteins and CYP24A1 may serve as surrogate markers for progesterone calcitriol treatment in ovarian and endometrial cancers of different histological types. Transl Cancer Res 2019;8:1423-37.

7. Schmitt M, Schewe M, Sacchetti A, et al. Paneth Cells Respond to Inflammation and Contribute to Tissue Regeneration by Acquiring Stem-like Features through SCF/c-Kit Signaling. Cell Rep 2018;24:2312-28.e7.

8. Yu S, Cao S, Hong S, et al. miR-3619-3p promotes papillary thyroid carcinoma progression via $\mathrm{Wnt} / \beta$-catenin pathway. Ann Transl Med 2019;7:643..

9. Golestaneh N, Beauchamp E, Fallen S, et al. Wnt signaling promotes proliferation and stemness regulation of spermatogonial stem/progenitor cells. Reproduction 2009;138:151-62.

10. ShamsEldeen AM, Ashour H, Shoukry HS, et al. Combined treatment with systemic resveratrol and resveratrol preconditioned mesenchymal stem cells, maximizes antifibrotic action in diabetic cardiomyopathy. J Cell Physiol 2019;234:10942-63.

11. Gu Q, Tian H, Zhang K, et al. Wnt5a/FZD4 Mediates the

Cite this article as: Yao L, Peng H, Xu Z, Shi L, Li Y, Dai Y. The effect of regulating the Wnt signaling pathway on the proliferation and differentiation of spermatogonial stem cells. Ann Transl Med 2020;8(16):1003. doi: 10.21037/atm-20-5321
Mechanical Stretch-Induced Osteogenic Differentiation of Bone Mesenchymal Stem Cells. Cell Physiol Biochem 2018;48:215-26.

12. Ibtisham F, Honaramooz A. Spermatogonial stem cells for in vitro spermatogenesis and in vivo restoration of fertility. Cells 2020;9:745.

13. Cai Y, Wang J, Zou K. The Progresses of spermatogonial stem cells sorting using fluorescence-activated cell sorting. Stem Cell Rev Rep 2020;16:94-102.

14. Ko K, Arauzo-Bravo MJ, Kim J, et al. Conversion of adult mouse unipotent germline stem cells into pluripotent stem cells. Nat Protoc 2010;5:921-8.

15. Sujit KM, Singh V, Trivedi S, et al. Increased DNA methylation in the spermatogenesis-associated (SPATA) genes correlates with infertility. Andrology 2020;8:602-9.

16. Zhao X, Yang HQ.(Progress on spermatogonial stem cells of large animals. Yi Chuan 2019;41:686-702.

17. Valdivia M, Castaneda-Zegarra S, Levano G, et al. Spermatogonial stem cells identified by molecular expression of PLZF, integrin beta1 and reactivity to Dolichos biflorus agglutinin in alpaca adult testes. Andrologia 2019;51:e13283.

18. Yeh JR, Zhang X, Nagano MC. Indirect effects of Wnt3a/ beta-catenin signalling support mouse spermatogonial stem cells in vitro. PLoS One 2012;7:e40002.

19. Jin $\mathrm{L}, \mathrm{Cao} \mathrm{Y}, \mathrm{Yu} \mathrm{G}$, et al. SFRP2 enhances the osteogenic differentiation of apical papilla stem cells by antagonizing the canonical WNT pathway. Cell Mol Biol Lett 2017;22:14.

20. Yu WZ, Chen XM, Niu WB, et al. Role of Wnt5a in the differentiation of human embryonic stem cells into endometrium-like cells. Int J Clin Exp Pathol 2015;8:5478-84.

21. He N, Wang $\mathrm{Y}$, Zhang $\mathrm{C}$, et al. Wnt signaling pathway regulates differentiation of chicken embryonic stem cells into spermatogonial stem cells via Wnt5a. J Cell Biochem 2018;119:1689-701.

(English Language Editor: J. Brown) 